\author{
() 3.Н. Джемилова ${ }^{1 *}$, И.И. Ситкин ${ }^{1}$, С.В. Сергеева' ${ }^{1}$, П.В. Грачев², К.Г. Линьков ${ }^{2}$, В.Б. Лощенов ${ }^{2}$, Г.Р. Галстян ${ }^{1}$
}

'Национальный медицинский исследовательский центр эндокринологии,

Москва ${ }^{2}$ Институт общей физики им. А.М. Прохорова, Москва

Описан клинический случай пациента с сахарным диабетом 2 типа (СД2) на стадии критической ишемии нижней конечности (КИНК) и ее разрешения после успешной реваскуляризации артерий нижней конечности (АНК). Диагноз КИНК был установлен на основании клинической картины, а также результатов неинвазивных методов диагностики ишемии нижней конечности. Особенностью случая является представление результатов нового метода оценки ишемии нижних конечностей - флуоресцентной ангиографии (ФАГ) в ближнем инфракрасном диапазоне (БИД) мягких тканей стопы в периоперационном периоде. Для оценки тяжести ишемии нижней конечности проводится анализ параметров ФАГ в БИД в различных зонах стопы. Предложены следующие параметры ФАГ в БИД: $\mathrm{T}_{\text {start }}$ (c) - время появления флуоресценции (I start юнит) в анализируемой области после внутривенного введения индоцианина зеленого (ИЦЗ); $\mathrm{T}_{\max }$ (с) - время достижения максимальной флуоресценции (I ${ }_{\max }$ юнит) после внутривенного введения ИЦ3; $\mathrm{T}_{\max }-$ $\mathrm{T}_{\text {start }}(\mathrm{c})$ - разница во времени достижения $\mathrm{I}_{\max }$ и $\mathrm{I}_{\text {start }}$. В данном клиническом случае показано сокращение времени достижения $\mathrm{I}_{\text {start' }} \mathrm{I}_{\text {max' }} \mathrm{T}_{\text {max }}-\mathrm{T}_{\text {start }}$ в различных анализируемых зонах стопы у пациента после успешной реваскуляризации AHK.

КЛЮЧЕВЫЕ СЛОВА: сахарный диабет; заболевания артерий нижних конечностей; критическая ишемия нижних конечностей; эндоваскулярное лечение; флуоресцентная ангиография в ближнем инфракрасном диапазоне; флуоресцентная ангиография с использованием индоцианина зеленого; синдром диабетической стопы; трофическая язва стопы

\title{
NEAR-INFRARED FLUORESCENCE IMAGING WITH INDOCYANINE GREEN IN DIABETIC PATIENT WITH CRITICAL LIMB ISCHEMIA: A CASE REPORT
}

Zera N. Dzhemilova1*, Ivan I. Sitkin', Svetlana V. Sergeeva', Pavel V. Grachev², Kirill G Linkov², Viktor B. Loshenov², Gagik R. Galstyan 1

'Endocrinology Research Centre, Moscow, Russia

${ }^{2}$ General Physics Institute of the Russian Academy of Sciences, Moscow, Russia

A case report of type 2 diabetic patient with critical limb ischemia (CLI) after successful endovascular revascularization is reported. The diagnosis of CLI was established according to clinical data and results of lower limb ischemia assessment by non-invasive methods. The unique feature of this case is presentation of results of the new method of lower limb ischemia assessment - fluorescent angiography in near infrared range using indocyanine green (ICG). Following parameters of fluorescent angiography in near infrared range are analyzed in different regions of interest: $\mathrm{T}_{\text {start }}$ (sec) - the time of fluorescence occurrence $\left(I_{\text {start }}\right.$ unit) in the analyzed area after intravenous administration of ICG; $T_{\max }$ (sec) - time to achieve maximum fluorescence $\left(I_{\max }\right.$, unit) after intravenous injection of ICG; $T_{\max }-T_{\text {start }}(\mathrm{sec})$ - the time difference between $I_{\max }$ and $I_{\text {start }}$. In this clinical case, the time of achievement $\mathrm{I}_{\text {start }} \mathrm{I}_{\max } \mathrm{T}_{\max }-\mathrm{T}_{\text {start }}$ in different regions of interest decreased after successful endovascular revascularization of lower limb arteries.

KEYWORDS: diabetes mellitus; peripheral artery disease; critical limb ischemia; endovascular treatment; near infrared fluorescence angiography; indocyanine green fluorescence angiography; diabetic foot; foot ulcer

По данным исследования ADVANCE, у 4,6\% пациентов с сахарным диабетом 2 типа (СД2) раневые дефекты или ампутации ниже колена были следствием заболевания артерий нижних конечностей (ЗАНК), из которых у 1,7 \% пациентов в связи с развитием критической ишемии нижней конечности (КИНК) выполнялась реваскуляризация артерий нижней конечности (АНК) с целью сохранения нижней конечности [1].

Скрининг на предмет наличия ЗАНК у пациентов с СД показан лицам в группе риска их развития. Инструментальное обследование данной категории пациентов включает оценку: лодыжечного артериального давле- ния (ЛАД), лодыжечно-плечевого индекса (ЛПИ); при необходимости пальце-плечевого индекса (ППИ), формы пульсовой волны на АНК, транскутанного напряжения кислорода $\left(\mathrm{TcpO}_{2}\right)$ мягких тканей голени и стопы; перфузионного давления кожи [2, 3, 4]. При декомпенсации хронической артериальной недостаточности (ХАН) вследствие ЗАНК развивается КИНК. К критериям постановки диагноза КИНК относятся клинические признаки, такие как постоянная боль в покое, требующая регулярного приема анальгетиков в течение 2 нед и более, и/или трофическая язва или гангрена пальцев или стопы, возникшие на фоне ХАН [2]. 
Однако в ряде случаев стандартные методы диагностики ЗАНК не позволяют оценить тяжесть хронической ишемии нижних конечностей (ХИНК) у пациентов с СД и ЗАНК, что обусловлено специфическим поражением артерий и трофическими изменениями тканей нижних конечностей. Применение ЛПИ ограничено при локализации раневого дефекта в области голени и стопы и бывает неинформативно при окклюзиях и кальцинозе тибиальных артерий. ППИ не применяется в случае трофических поражений пальцев стопы или их отсутствии. $\mathrm{TcpO}_{2}$ имеет преимущества перед вышеуказанными методами ввиду отсутствия влияния окклюзий, кальциноза артерий на методику проведения исследования. Электроды датчика ТсрО 2 можно устанавливать на свободном от раневых дефектов участке кожи. Однако интерпретация результатов ТсрО 2 с целью оценки тяжести ХИНК затруднена при наличии воспаления, отека мягких тканей стопы и т.д. [5].

Вышеуказанные обстоятельства диктуют необходимость поиска новых методов оценки ХИНК у пациентов с СД и ЗАНК. Метод флуоресцентной ангиографии в ближнем инфракрасном диапазоне (ФАГ в БИД) активно используется в различных отраслях медицины [6] и предлагается как один из дополнительных методов оценки ишемии нижних конечностей у лиц с СД и ЗАНК $[7,8]$.

Подробное описание метода и результатов пилотного исследования ФАГ в БИД было опубликовано ранее [9]. Флуоресцентный ангиограф разработан на базе лаборатории лазерной биоспектроскопии Института общей физики им. А.М. Прохорова РАН (ЗАО «БИОСПЕК», Россия). Для получения флуоресценции внутривенно вводился флуорофор индоцианин зеленый (ИЦЗ), диодный лазер с источником длины волны 785 нм направлялся на интересующую область исследования (например, мягкие ткани стопы); камера с объективом и набором фильтров фиксировала результаты ФАГ и передавала на персональный компьютер, где проводился анализ полученных данных. Продолжительность исследования составляла 15 мин. По предлагаемому протоколу оценивались сле-

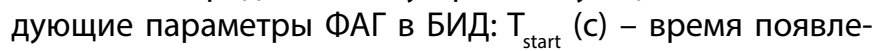
ния флуоресценции ( start юнит) в анализируемой области после внутривенного введения ИЦ3; $\mathrm{T}_{\max }$ (c) - время достижения максимальной флуоресценции (I max' $^{\prime}$ юнит) после внутривенного введения ИЦ3; $\mathrm{T}_{\max }-\mathrm{T}_{\text {start }}(\mathrm{c})$ - разница во времени достижения $\mathrm{I}_{\max }$ и $\mathrm{I}_{\text {start }}$.

\section{ОПИСАНИЕ СЛУЧАЯ}

Пациент Б., 69 лет, поступил в отделение «Диабетическая стопа» ФГБУ «НМИЦ эндокринологии» Минздрава России в феврале 2018 г. Ведущие жалобы при госпитализации на боль в IV, V пальцах левой стопы, почернение IV пальца левой стопы.

\section{Анамнез заболевания; результаты проведенного \\ обследования}

СД2 диагностирован в 2012 г. при обследовании пациента по поводу язвенного дефекта правой голени. Рекомендована терапия с ограничением приема легкоусвояемых углеводов, глимепирид 1 мг утром. При ежедневном самоконтроле гликемии натощак 5-7 ммоль/л, редко - эпизоды повышения гликемии до 11 ммоль/л, гипогликемии в течение дня 1-2 раза в мес. В феврале 2017 при госпитализации в отделении «Диабетическая стопа» ФГБУ «Эндокринологический научный центр» проведена коррекция терапии в связи с низконормальными показателями гликемии: глимепирид заменен на вилдаглиптин 50 мг утром. Гликированный гемоглобин от 27.02.2018 7,7\%.

Ежегодно проводилось обследование пациента на предмет наличия микро- и макрососудистых осложнений СД. Диабетическая нефропатия отсутствует. В 2012 г. диагностирована непролиферативная диабетическая ретинопатия обоих глаз, на настоящий момент состояние глазного дна без отрицательной динамики. В 2012 г. выявлен стеноз правой внутренней сонной артерии (BCA) 90\%. Выполнена эверсионная каротидная эндартерэктомия правой ВСА. В 2013 г. диагностирован инфаркт миокарда. Интервенционных вмешательств на коронарных артериях не проводилось. В феврале 2017 г. диагностирована постоянная форма фибрилляций предсердий. Проводится кардиопротективная терапия. На основании клинических симптомов и результатов обследования диагностированы: Сахарный диабет 2 типа. Дистальная диабетическая полинейропатия 3 ст. Облитерирующий атеросклероз артерий нижних конечностей. Гангрена IV пальца левой стопы, WIFI 2-3-2. Состояние после экзартикуляции I-III пальцев левой стопы. ИБС: постинфарктный кардиосклероз. Постоянная форма фибрилляции предсердий CHA2DS2VASc 4 балла. Хроническая сердечная недостаточность 2а ст. II ФК по NYHA. Нестенозирующий атеросклероз брахиоцефальных артерий.

Рецидив КИНК:

незаживающие

Боли при ходьбе, дискомфорт КИНК: гангрена в ногах I, II пальцев стопы раны на месте ампутации I, II пальцев $\begin{array}{cc} & \text { Гангрена } \\ \text { Остеомиелит } & \text { IV пальца, } \\ \text { III пальца } & \text { рецидив КИНK }\end{array}$

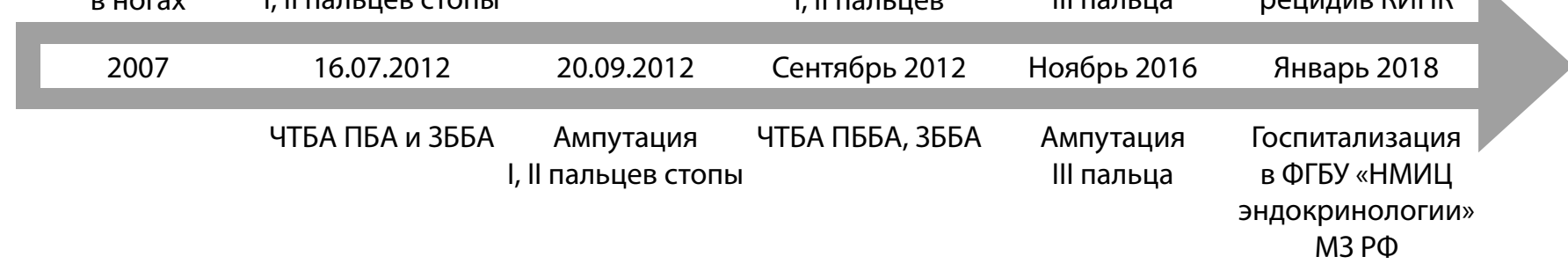

Рис. 1. Динамика клинических изменений и интервенционных вмешательств на левой нижней конечности пациента Б. Примечания: ПБА - поверхностная бедренная артерия; ПББА - передняя большеберцовая артерия; ЗББА - задняя большеберцовая артерия; КИНК - критическая ишемия нижней конечности, ЧТБА - чрескожная транслюминальная баллонная ангиопластика. 
Таблица 1. Результаты лодыжечного артериального давления, лодыжечно-плечевого индекса, транскутанного напряжения кислорода у пациента Б. на стадии критической ишемии нижних конечностей

\begin{tabular}{cccc}
\hline & Дистальный сегмент ПББА & Дистальный сегмент 3ББА & $\begin{array}{c}\text { Уровень значений при КИНК } \\
\text { [2] }\end{array}$ \\
\hline ЛАД & 40 & 30 & $<70$ \\
ЛПИ & 0,28 & 0,2 & $<0,5$
\end{tabular}

Кожа тыльной поверхности стопы Кожа у медиальной лодыжки

$\mathrm{TcpO}_{2}$, мм рт. ст.

7

$<25$
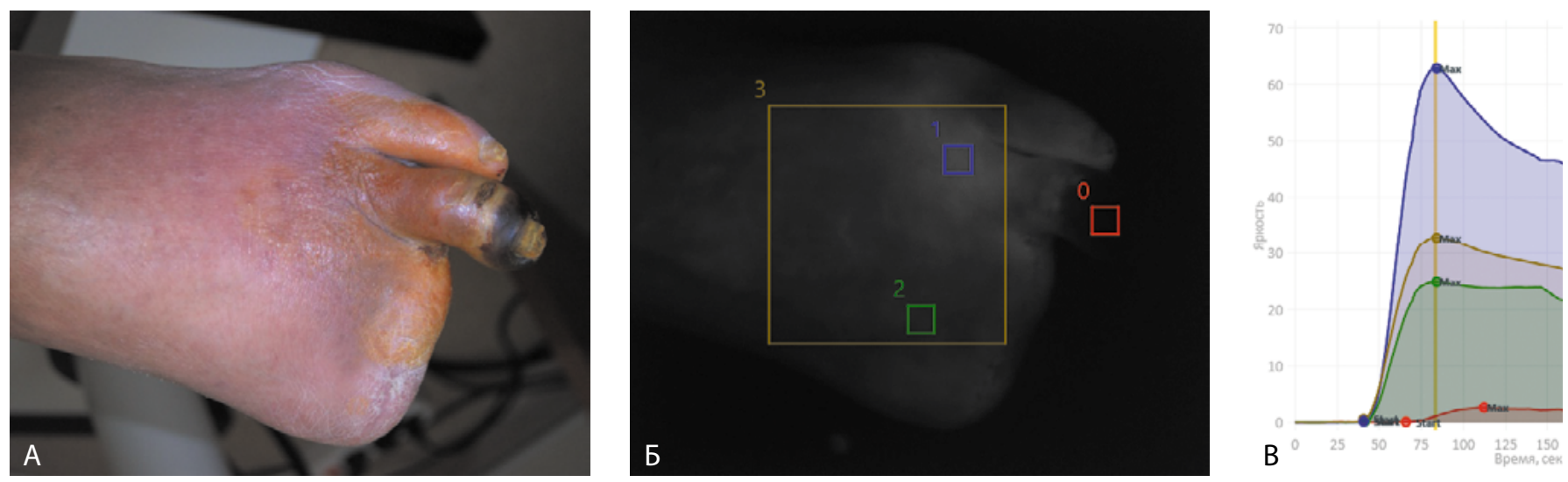

Рис. 2. Результат флуоресцентной ангиографии (ФАГ) в ближнем инфракрасном диапазоне (БИД) левой стопы. А - вид стопы на момент исследования. Б - вид стопы в ходе проведения ФАГ в БИД. Области оценки параметров ФАГ в БИД: 0 - область минимальной флуоресценции; 1 - область максимальной флуоресценции; 2 - область 1-го межпальцевого промежутка; 3 - область исследования большей части стопы. В - диаграмма ФАГ в БИД в зонах интереса 0-3. $\mathrm{T}_{\text {start }}$ (c) - время появления флуоресценции (I $\mathrm{I}_{\text {start }}$ Юнит) в исследуемой области после внутривенного введения индоцианина зеленого (ИЦЗ); $\mathrm{T}_{\text {max }}$ (c) время достижения максимальной флуоресценции (I ${ }_{\text {max }}$ юнит) после внутривенного введения ИЦз; $\mathrm{T}_{\max }-\mathrm{T}_{\text {start }}$ (c) - разница во времени достижения $\mathrm{I}_{\max } n \mathrm{I}_{\text {start: }}$

Таблица 2. Результаты флуоресцентной ангиографии в ближнем инфракрасном диапазоне левой стопы у пациента Б. на стадии критической ишемии нижних конечностей

\begin{tabular}{lccccc}
\hline & $\mathbf{T}_{\text {start }} \mathbf{c}$ & $\mathbf{I}_{\text {start }} \mathbf{C}$ & $\mathbf{T}_{\text {max }^{\prime}} \mathbf{C}$ & $\mathbf{I}_{\text {max }^{\prime}} \mathbf{C}$ & $\mathbf{T}_{\max }-\mathbf{T}_{\text {start' }} \mathbf{C}$ \\
\hline 0_Область минимальной флуоресценции & 66 & 0,14 & 11,15 & 2,7 & 46,15 \\
1_Область максимальной флуоресценции & 41 & 0,23 & 84,15 & 62,95 & 43,15 \\
2_Область 1-го межпальцевого промежутка & 42 & 0,31 & 84,15 & 24,94 & 42,15 \\
3_Область исследования большей части стопы & 41 & 0,64 & 84,15 & 32,86 & 43,15 \\
\hline
\end{tabular}

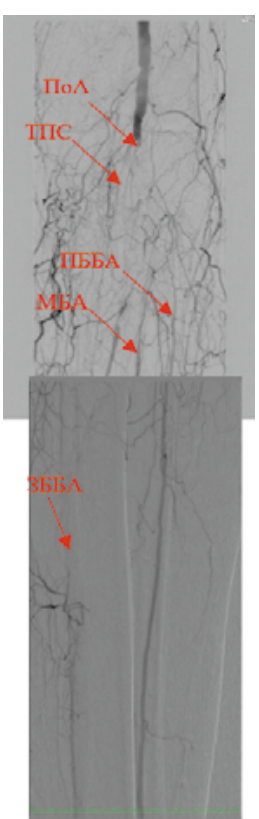

Рис. 3. Результат диагностической субтракционной ангиографии пациента Б., 69 лет, с сахарным диабетом и критической ишемией нижних конечностей. Выявлены окклюзии в дистальном сегменте подколенной артерии (ПоА); окклюзия тибиоперонеального ствола (ТПС), малоберцовой артерии (МБА), задней большеберцовой артерии (ЗББА), передней большеберцовой артерии (ПББА).
Непролиферативная форма диабетической ретинопатии обоих глаз. Факоэмульсификация катаракты обоих глаз с имплантацией интраокулярной линзы.

Терапия пациента на момент поступления в ФГБУ «НМИЦ эндокринологии» Минздрава России: Вилдаглиптин (Галвус) 50 мг 2 р/сут, Гликлазид МВ 30 мг утром, Метопролол 25 мг 2 р/сут, Эналаприл 2,5 мг утром, Клопидогрел 75 мг утром, Аторвастатин 10 мг на ночь, Аллопуринол 100 мг вечером, Дабигатран 150 мг 2 р/сут.

С февраля 2012 г. в связи с развитием КИНК правой нижней конечности (незаживающие раневые дефекты) неоднократно проводилась чрескожная транслюминальная баллонная ангиопластика (ЧТБА) артерий правой нижней конечности, хирургические вмешательства на стопе. Локальный статус на момент поступления: состояние после малой ампутации в объеме трансметатарзальной резекции правой стопы. Ортопедическое пособие: индивидуальная сложная ортопедическая обувь с индивидуальной стелькой.

Динамика клинических изменений и интервенционных вмешательств на левой нижней конечности представлена на рис. 1.

Локальный статус левой нижней конечности на момент поступления в отделение диабетической стопы: 


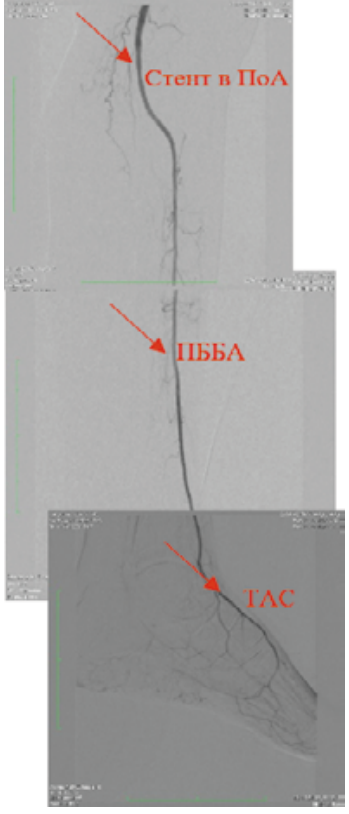

Рис. 4. Результат проведенной чрескожной транслюминальной баллонной ангиопластики и стентирования подколенной артерии (ПоА), баллонной ангиопластики передней большеберцовой артерии (ПББА) на всем протяжении, включая подошвенную артериальную дугу. ТАС тыльная артерия стопы. отек и гиперемия тыльной поверхности стопы, некроз IV пальца WIFI 2-3-2; состояние после экзартикуляции I-III пальцев стопы.

\section{Результат инструментального обследования левой} нижней конечности, проведенного в отделении

\section{Оченка тяжести ишемии конечности}

Кровоток на тыльной и задней большеберцовой артериях стопы (минидоп) - коллатеральный. Результаты ЛАД, ЛПИ, ТсрО п представлены в табл. 1.

Результаты параметров ФАГ в БИД левой стопы указаны на рис. 2, в табл. 2.

Результаты визуализирующих методов оченки артериального кровотока артерий левой нижней конечности

Ультразвуковое дуплексное сканирование артерий: гемодинамически-незначимые стенозы (до 50\%) общей бедренной артерии, глубокой артерии бедра, поверхностной бедренной артерии. Фрагментарные окклюзии подколенной артерии (ПоА), тибиоперонеального ствола (ТПС), малоберцовой артерии (МБА), передней большеберцовой артерии (ПББА), задней большеберцовой артерии (ЗББА), тыльной артерии стопы (ТАС).

Таблица 3. Результаты лодыжечного артериального давления, лодыжечно-плечевого индекса, транскутанного напряжения кислорода у пациента Б. после чрескожной транслюминальной баллонной ангиопластики артерий левой нижней конечности

\begin{tabular}{|c|c|c|c|}
\hline & Дистальный сегмент ПББА & Дистальный сегмент ЗББА & $\begin{array}{c}\text { Уровень значений при разре- } \\
\text { шении КИНК [2] }\end{array}$ \\
\hline ЛАД, мм рт. ст. & 100 & 100 & $>70$ \\
\hline \multirow[t]{2}{*}{ лПи } & 0,71 & 0,7 & $\geq 0,5$ \\
\hline & Кожа тыльной поверхности стопы & Кожа у медиальной лодыжки & \\
\hline $\mathrm{TcpO}_{2}$, мм рт.ст. & 43 & 38 & $\geq 25$ \\
\hline
\end{tabular}

A

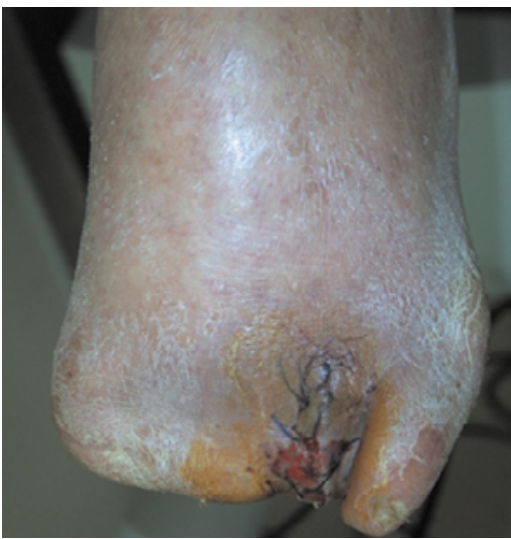

Б

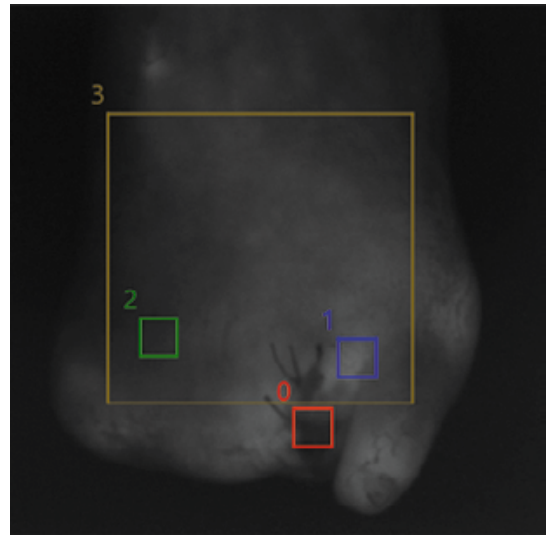

B

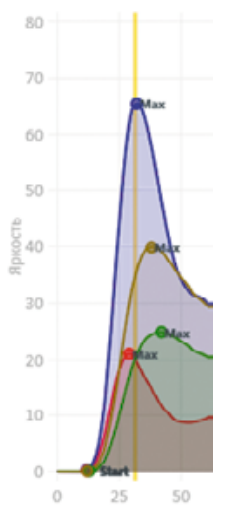

Рис. 5. Результат параметров флуоресцентной ангиографии (ФАГ) в ближнем инфракрасном диапазоне (БИД) левой стопы в послеоперационном периоде.. А - вид стопы на момент исследования. Б - вид стопы в процессе проведения ФАГ в БИД. Области оценки параметров ФАГ в БИД: 0 - область минимальной флуоресценции; 1 - область максимальной флуоресценции; 2 - область 1-го межпальцевого промежутка, 3 - область исследования большей части стопы. В - диаграмма ФАГ в

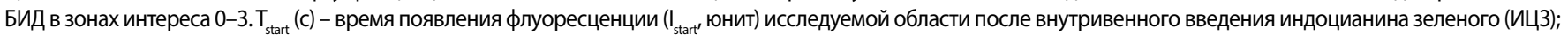
$\mathrm{T}_{\max }(\mathrm{c})$ - время достижения максимальной флуоресценции (I ${ }_{\max }$ Юнит) после внутривенного введения ИЦЗ; $\mathrm{T}_{\max }-\mathrm{T}_{\text {start }}$ (c) - разница во времени достижения $\mathrm{I}_{\max }$ и $\mathrm{I}_{\text {start }}$

Таблица 4. Результаты флуоресцентной ангиографии в ближнем инфракрасном диапазоне левой стопы у пациента Б. после чрескожной транслюминальной баллонной ангиопластики артерий левой нижней конечности

\begin{tabular}{|c|c|c|c|c|c|}
\hline & $\mathbf{T}_{\text {start }} \mathbf{C}$ & $I_{\text {start }}, C$ & $T_{\text {max }}, \mathbf{C}$ & $I_{\max }, C$ & $\mathbf{T}_{\max }-\mathbf{T}_{\text {start }} \mathbf{C}$ \\
\hline 0_Область минимальной флуоресценции & 12 & 0,37 & 32 & 65,5 & 20 \\
\hline 1_Область максимальной флуоресценции & 13 & 0,2 & 29 & 21,1 & 16 \\
\hline 2_Область 1-го межпальцевого промежутка & 12 & 0,12 & 41 & 24,9 & 29 \\
\hline 3_Область исследования большей части стопы & 12 & 0,12 & 38 & 39,9 & 26 \\
\hline
\end{tabular}


Диагностическая селективная ангиография: окклюзия ПоА ниже уровня щели коленного сустава, окклюзия ТПС, окклюзия МБА и ЗББА на всем протяжении, ПББА (рис. 3).

Лечение критической ишемии левой нижней конечности

Первым этапом хирургического лечения выполнены ЧТБА и стентирование ПоА, баллонная ангиопластика (БА) ПББА на всем протяжении, включая подошвенную артериальную дугу. При контрольной ангиографии свободное контрастирование включая дистальное русло на стопе (рис. 4).

Следующим этапом проведено хирургическое лечение в объеме экзартикуляции IV пальца стопы в проксимальном межфаланговом суставе.

Результаты послеоперационной оценки тяжести ишемии левой нижней конечности представлены в табл. 3.

Результаты параметров ФАГ в БИД левой стопы в послеоперационном периоде указаны на рис. 5, в табл. 4.

В периоперационном периоде проводились ежедневные перевязки стопы с растворами жидких антисептиков, антибактериальная терапия, разгрузка левой нижней конечности. Достигнут клинический эффект лечения КИНК левой нижней конечности: отсутствие боли в стопе, заживление раневого дефекта. Пациент выписан с рекомендациями по лечению и динамическому наблюдению.

\section{ОБСУЖДЕНИЕ}

Основной целью текущей госпитализации являлось лечение рецидива КИНК левой нижней конечности, формирующей высокий риск ампутации нижней конечности согласно стратификации рисков по классификации WIFI. Диагноз КИНК левой нижней конечности был установлен на основании клинического обследования (боли в стопе, гангрена II пальца левой стопы), тяжесть ишемии подтверждена неинвазивными методами диагностики: уровень ЛАД менее 40 мм рт.ст., ЛПИ менее 0,28, уровень $\mathrm{TcpO}_{2}-7$ мм рт.ст. По данным ФАГ в БИД определены временные параметры старта флуоресценции и достижения максимального уровня флуоресценции после внутривенного введения ИЦЗ в различных зонах интереса на стопе (см. рис. 2, см. табл. 2) у пациента на стадии КИНК. После успешной реваскуляризации АНК подтверждено разрешение КИНК согласно результатам неинвазивных методов диагностики ишемии с достижением оптимального уровня оксигенации тканей стопы. По данным результатов проведенного ФАГ в БИД в после- операционном периоде выявлено сокращение времени появления и достижения максимальной флуоресценции ИЦЗ после его внутривенного введения (см. рис. 5, табл. 4). Подобные результаты проведенных ФАГ в БИД у пациентов с ЗАНК наблюдались в исследованиях зарубежных коллег $[7,8]$.

ФАГ в БИД дает возможность оценить количественные (временные) и качественные (уровень флуоресценции) параметры кровотока различных участков мягких тканей стопы. На методику проведения исследования не влияло наличие окклюзий и кальциноза артерий нижних конечностей, отека стопы. В режиме реального времени можно было оценить интенсивность флуоресценции мягких тканей стопы, что также может иметь преимущество в использовании данного метода в ходе реваскуляризации АНК. Одним из условно отрицательных моментов данной методики можно считать необходимость внутривенного введения флуорофора ИЦЗ, являющегося трикарбоновым красителем, позволяющим получать изображения при воздействии света в БИД. В 1956 г. ИЦЗ был одобрен Управлением по санитарному надзору за качеством пищевых продуктов и медикаментов США

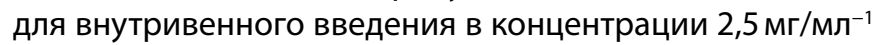
с максимально допустимой дозой введения для взрослого человека 25 мг [10].

\section{ЗАКЛЮЧЕНИЕ}

ФАГ в БИД может рассматриваться как перспективный метод, существенным образом дополняющий имеющиеся методы оценки тяжести ишемии у пациентов с ЗАНК. Вместе с этим требуются дальнейшие исследования с включением репрезентативной выборки пациентов с СД для оценки прогностической значимости ФАГ в БИД в заживлении раневых дефектов и определения показаний для реваскуляризации АНК у пациентов с КИНК.

\section{ДОПОЛНИТЕЛЬНАЯ ИНФОРМАЦИЯ}

Согласие пациента. Пациент добровольно подписал информированное согласие на публикацию персональной медицинской информации в обезличенной форме в журнале «Сахарный диабет».

Конфликт интересов. Авторы декларируют отсутствие явных и потенциальных конфликтов интересов, связанных с публикацией настоящей статьи.

\section{СПИСОК ЛИТЕРАТУРЫ | REFERENCES}

1. Mohammedi K, Woodward M, Hirakawa Y, et al. Presentations of major peripheral arterial disease and risk of major outcomes in patients with type 2 diabetes: results from the ADVANCE-ON study. Cardiovasc Diabetol. 2016;15(1):129. doi: 10.1186/s12933-016-0446-х

2. Дедов И.И., Шестакова М.В., Майоров А.Ю., и др. Алгоритмы специализированной медицинской помощи больным сахарным диабетом / Под ред. Дедова И.И., Шестаковой М.В., Майорова А.Ю. - 8-й выпуск // Сахарный диабет. - 2017. - Т. 20. - №1S. C. 1-121. [Dedov II, Shestakova MV, Mayorov AY, et al. Dedov II, Shestakova MV, Mayorov AY, et al. Standards of specialized diabetes care. 8th edition. Diabetes mellitus. 2017;20(1S):1-121. (In Russ.)] doi: 10.14341/DM20171S8

3. Brownrigg JR, Hinchliffe RJ, Apelqvist J, et al. Effectiveness of bedside investigations to diagnose peripheral artery disease among people with diabetes mellitus: a systematic review. Diabetes Metab Res Rev. 2016;32 Suppl 1:119-127. doi: 10.1002/dmrr.2703

4. Aboyans V, Ricco JB, Bartelink MEL, et al. 2017 ESC Guidelines on the Diagnosis and Treatment of Peripheral Arterial Diseases, in collaboration with the European Society for Vascular Surgery (ESVS): Document covering atherosclerotic disease of extracranial carotid and vertebral, 
mesenteric, renal, upper and lower extremity arteriesEndorsed by: the European Stroke Organization (ESO)The Task Force for the Diagnosis and Treatment of Peripheral Arterial Diseases of the European Society of Cardiology (ESC) and of the European Society for Vascular Surgery (ESVS). Eur Heart J. 2018;39(9):763-816. doi: 10.1093/eurheartj/ehx095

5. Бондаренко О.Н., Аюбова НЛ., Галстян Г.Р., Дедов И.И. Транскутанная оксиметрия в динамическом наблюдении за пациентами с сахарным диабетом и критической ишемией нижних конечностей // Сахарный duaбem. - 2013. - T. 16. - №1. - C. 33-42. [Bondarenko ON, Ayubova NL, Galstyan GR, Dedov II. Transcutaneous oximetry monitoring in patients with type 2 diabetes mellitus and critical limb ischemia. Diabetes mellitus. 2013;16(1):33-42. (In Russ.)] doi: 10.14341/2072-0351-3594

6. Alander JT, Kaartinen I, Laakso A, et al. A review of indocyanine green fluorescent imaging in surgery. Int J Biomed Imaging 2012;2012:940585. doi: 10.1155/2012/940585

7. Igari K, Kudo T, Toyofuku T, et al. Quantitative evaluation of the outcomes of revascularization procedures for peripheral arterial disease using indocyanine green angiography. Eur J Vasc Endovasc Surg. 2013;46(4):460-465. doi: 10.1016/j.ejvs.2013.07.016

8. Braun JD, Trinidad-Hernandez M, Perry D, et al. Early quantitative evaluation of indocyanine green angiography in patients with critical limb ischemia. J Vasc Surg. 2013;57(5):1213-1218. doi: 10.1016/j.jvs.2012.10.113

9. Абдульвапова 3.Н., Грачев П.В., Артемова Е.В., и др. Оценка состояния кровотока нижних конечностей у пациентов с сахарным диабетом методом флуоресцентной ангиографии в ближнем инфракрасном диапазоне // Biomedical Photonics. - 2017. - T. 6. №1. - C. 4-11. [Abdulvapova ZN, Grachev PV, Artemova EV, et al. Near infrared imaging for angiography in diabetic patients with peripheral artery disease. Biomedical Photonics. 2017;6(1):4-11. (In Russ.)] doi: 10.24931/2413-9432-2017-6-1-4-11

10. Engel E, Schraml R, Maisch T, et al. Light-induced decomposition of indocyanine green. Invest Ophthalmol Vis Sci. 2008;49(5):1777-1783. doi: 10.1167/iovs.07-0911

\section{ИНФОРМАЦИЯ ОБ АВТОРАХ [AUTHORS INFO]}

*Джемилова Зера Нусредовна, н.c. [Zera N. Dzhemilova, MD, research associate]; адрес: 117036, Москва, ул. Дм. Ульянова, д. 11 [address: 11, Dm.Ulyanova street, Moscow, 117036 Russian Federation]; ORCID: http://orcid.org/0000-0002-1129-8995; eLibrary SPIN: 4455-5667; e-mail: zera1987@mail.ru

Ситкин Иван Иванович, к.M.н., С.H.c. [lvan I. Sitkin, MD, PhD, senior research associate]; ORCID: http://orcid.org/0000-0002-2175-3170; eLibrary SPIN: 9779-3780; e-mail: sitkin_ivan@rambler.ru Сергеева Светлана Викторовна, врач-эндокринолог [Svetlana V. Sergeeva, MD]; ORCID: https://orcid.org/0000-0002-8722-5434; e-mail: svetaser@bk.ru Грачев Павел Вячеславович, H.c. [Pavel V. Grachev, research associate]; ORCID: http://orcid.org/0000-0003-4333-1830; eLibrary SPIN: 4739-1232; e-mail: p.v.grachev@gmail.com

Линьков Кирилл Геннадиевич, К.т.Н., С.Н.C. [Kirill G. Linkov, PhD in Technology Sciences, senior research associate]; ORCID: http://orcid.org/0000-0003-0218-5443; eLibrary SPIN: 4046-2022; e-mail: rkir123@gmail.com

Лощенов Виктор Борисович, д.М.Н., профессор [Viktor B. Loshenov, MD, PhD, Professor]; ORCID: http://orcid.org/0000-0002-0507-2367; eLibrary SPIN: 7740-7676; e-mail: loschenov@mail.ru Галстян Гагик Радикович, д.М.H., профессор [Gagik R. Galstyan, MD, PhD, Professor]; ORCID: http://orcid.org/0000-0001-6581-4521; eLibrary SPIN: 9815-7509; e-mail: galstyangagik964@gmail.com

\section{ЦИТИРОВАТЬ:}

Джемилова 3.Н., Ситкин И.И., Сергеева С.В., Грачев П.В., Линьков К.Г., Лощенов В.Б., Галстян Г.Р. Применение метода флуоресцентной ангиографии в ближнем инфракрасном диапазоне у пациента с сахарным диабетом и критической ишемией нижней конечности // Сахарный диабет. — 2018. — T. 21. — №4. — C. 319-324. doi: 10.14341/DM9764

\section{TO CITE THIS ARTICLE:}

Dzhemilova ZN, Sitkin II, Sergeeva SV, Grachev PV, Linkov KG, Loshenov VB, Galstyan GR. Near-infrared fluorescence imaging with indocyanine green in diabetic patient with critical limb ischemia: a case report. Diabetes Mellitus. 2018;21(4):319-324. doi: 10.14341/DM9764 